\title{
Amicable Numbers and Their Distribution
}

\author{
By Paul Bratley, Fred Lunnon and John McKay
}

\begin{abstract}
All the amicable pairs whose smaller member is less than $10^{8}$ are now known. Using this data a conjecture is made about the distribution of amicable pairs. A result on "crowds" (sociable numbers of order 3 ) is also given.
\end{abstract}

In another paper in this issue of Math. Comp. [1] Cohen gives a list of all the pairs of amicable numbers whose smaller member lies between $10^{7}$ and $10^{8}$. The same list was computed independently by us on various machines; namely, the KDF 9 at Edinburgh University, Atlas at the Science Research Council's Chilton Laboratory, the London University CDC 6600, and the CDC 6400 at the University of Montreal. The results agree with those obtained by Cohen.

During the first part of the computation "crowds" (sociable numbers of order 3) were also sought: an exhaustive search showed that there exists no crowd whose smallest member is less than $5 \times 10^{7}$.

Let $A(n)$ denote the number of amicable pairs whose smaller member does not exceed $n$, and let $\Pi(n)$ be the number of primes which do not exceed $n$. In an unpublished paper [2] Lee suggests that $A(n)$ is proportional to $\Pi\left(n^{1 / 2}\right)$. Since from the prime number theorem

$$
\Pi\left(n^{1 / 2}\right) \sim \frac{n^{1 / 2}}{\ln \left(n^{1 / 2}\right)}=\frac{2 n^{1 / 2}}{\ln (n)}
$$

we compared $A(n)$ with $n^{1 / 2} / \ln (n)$. The curved line in Fig. 1 illustrates this comparison. The straight line in the same figure is a least squares fit to the curve, added as a guide to the eye. The slope of the line is 0.445 and its intercept on the vertical axis is 13.367 .

The following conclusions may be drawn from the figure:

(i) $A(n)$ is approximately proportional to $n^{1 / 2} / \ln (n)$ for $n<10^{8}$;

(ii) however, the curve is definitely concave downwards, which suggests that $A(n)$ may really be of a lower order;

(iii) in any case, it does appear that

$$
A(n) / n^{1 / 2} \rightarrow 0,
$$

which is stronger than Erdös's theorem [3] that

$$
A(n) / n \rightarrow 0 \text {. }
$$

It does not seem reasonable to draw more than these tentative conclusions from the data at present available. An extension of the exhaustive search for amicable

Received September 3, 1969, revised September 29, 1969.

AMS Subject Classifications. Primary 10 05, 10 42, $1043,1063$.

Key Words and Phrases. Amicable numbers, sociable numbers, aliquot series. 
numbers, combined with an analytic attack, might allow more satisfactory results to be obtained.

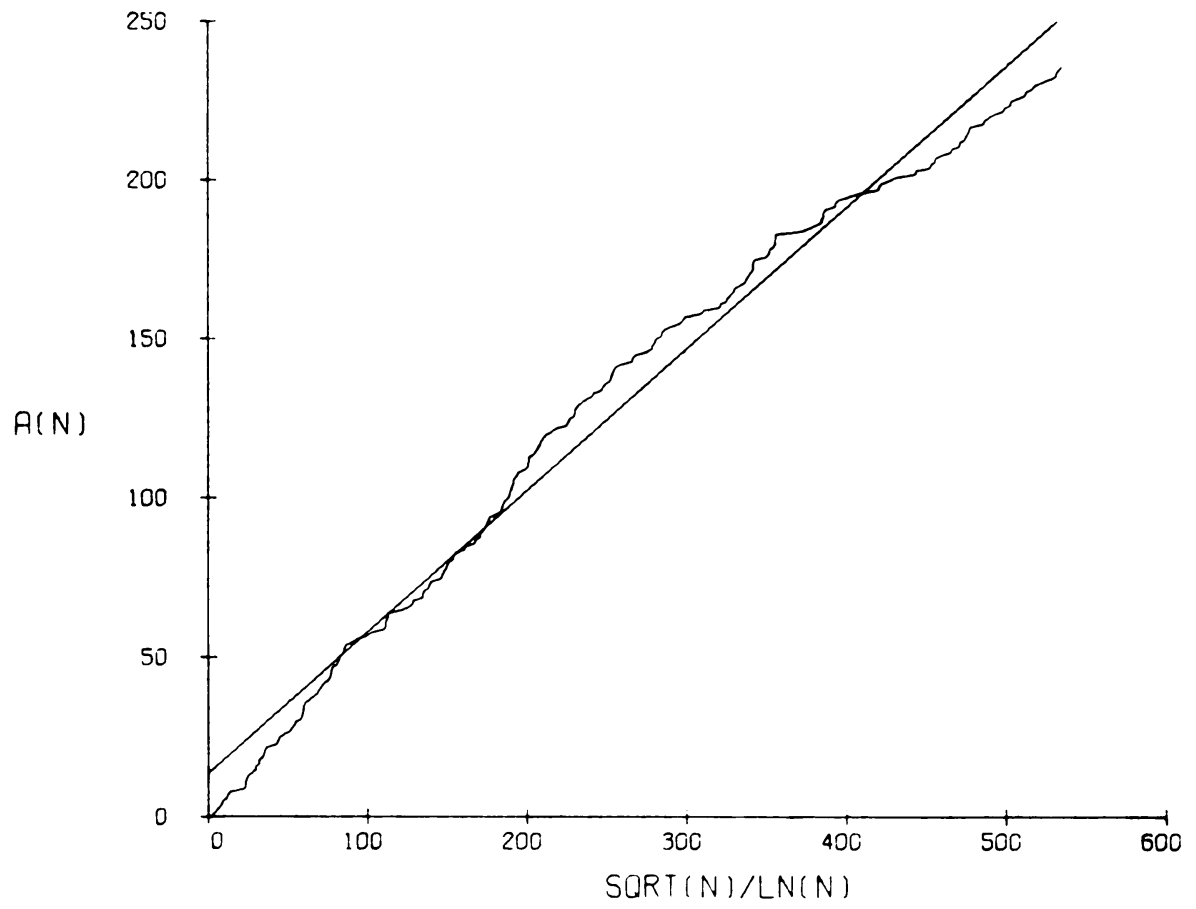

Figure 1

Département d'Informatique

Université de Montréal

Montréal, Québec, Canada

Atlas Computer Laboratory

Chilton, Didcot, England

1. Henri Cohen, "On amicable and sociable numbers," Math. Comp., v. 24, 1970, pp. 423-429.

2. Elvin J. LEE, "A note on the distribution of amicable pairs". (Unpublished.)

3. Paul Erdös, "On amicable numbers," Publ. Math. Debrecen, v. 4, 1955, pp. 108-111. MR 16, 998. 